Böhme, Thomas; Gerlach, Tobias; Stiebitz, Michael:

\title{
Ordered and linked chordal graphs
}

URN: $\quad$ urn:nbn:de:gbv:Ilm1-2020200158

\begin{tabular}{ll}
\hline Original published in: & $\begin{array}{l}\text { Discussiones mathematicae. Graph theory / Uniwersytet Zielonogórski, } \\
\text { Wydział Matematyki, Informatyki i Ekonometrii. - Warsaw : De Gruyter } \\
\end{array}$ \\
& Open. - 28 (2008), 2, p. 367-373. \\
& 2008 \\
Original published: & $2083-5892$ \\
ISSN: & $10.7151 /$ dmgt.1412 \\
DOI: & $2020-01-17]$ \\
[Visited: & \\
\hline & $\begin{array}{l}\text { This work is licensed under a Creative Commons Attribution- } \\
\text { NonCommercial-NoDerivatives 3.0 Unported license. } \\
\text { To view a copy of this license, visit } \\
\text { http://creativecommons.org/licenses/BY-NC-ND/3.0/ }\end{array}$ \\
\hline
\end{tabular}


Discussiones Mathematicae

Graph Theory 28 (2008) 367-373

Note

\title{
ORDERED AND LINKED CHORDAL GRAPHS
}

\author{
Thomas Böhme, Tobias Gerlach \\ AND \\ Michael Stiebitz \\ Institut für Mathematik \\ Technische Universität Ilmenau \\ Ilmenau, Germany \\ e-mail: tboehme@theoinf.tu-ilmenau.de \\ e-mail: tobias.gerlach@tu-ilmenau.de \\ e-mail: stieb@mathematik.tu-ilmenau.de
}

\begin{abstract}
A graph $G$ is called $k$-ordered if for every sequence of $k$ distinct vertices there is a cycle traversing these vertices in the given order. In the present paper we consider two novel generalizations of this concept, $k$-vertex-edge-ordered and strongly $k$-vertex-edge-ordered. We prove the following results for a chordal graph $G$ :

(a) $G$ is $(2 k-3)$-connected if and only if it is $k$-vertex-edge-ordered $(k \geq 3)$.

(b) $G$ is $(2 k-1)$-connected if and only if it is strongly $k$-vertex-edgeordered $(k \geq 2)$.

(c) $G$ is $k$-linked if and only if it is $(2 k-1)$-connected.
\end{abstract}

Keywords: paths and cycles, connectivity, chordal graphs.

2000 Mathematics Subject Classification: 05C38, 05 C40.

\section{Introduction AND Results}

All graphs considered in this paper are finite, undirected, and simple, i.e., without loops or multiple edges. For terminology not defined here we refer to [2]. A graph is chordal if it contains no induced cycles other 
than triangles, and it is called $k$-linked if for every set of $k$ distinct pairs $L=\left\{\left(s_{0}, t_{0}\right), \ldots,\left(s_{k-1}, t_{k-1}\right)\right\}$ of vertices it contains $k$ internally disjoint paths $P_{0}, \ldots, P_{k-1}$ such that $P_{i}$ links $s_{i}$ to $t_{i}$ for all $i \in\{0, \ldots, k-1\}$. We shall call the subgraph of $G$ formed by the union of $P_{0}, \ldots, P_{k-1}$ an $L$ linkage. Jung [5] and, independently, Larman and Mani [6] proved that for every $k$ there is an (minimal) $f(k)$ such that every $f(k)$-connected graph is $k$-linked. Bollobás and Thomason [1] showed that $f(k) \leq 22 k$. Recently, it was proved by Thomas and Wollan [8] that $f(k) \leq 10 k$. Our second result, Theorem 1.2 below, shows that for the special case of chordal graphs the precise value of $f(k)$ is $2 k-1$.

A graph is called $k$-ordered if for every sequence $\left(v_{0}, \ldots, v_{k-1}\right)$ of $k$ distinct vertices there is a cycle of $G$ that contains $v_{0}, \ldots, v_{k-1}$ in the given order. This concept was introduced by Ng and Schultz [7], and a survey of results on $k$-ordered graphs is given in [4]. It is easy to see that being $k$ linked implies being $k$-ordered. We generalize the concept of $k$-orderability as follows. Let $T=\left(a_{0}, \ldots, a_{k-1}\right)$ be a sequence of $k$ distinct vertices and/or edges, and let $V(T)$ and $E(T)$ denote the sets of vertices and edges in $T$, respectively. Let $W(T)$ denote the set of all vertices that are either contained in $T$ or incident to an edge in $T$. A $T$-cycle is a cycle in $G$ that contains $a_{0}, \ldots, a_{k-1}$ in the given order. The sequence $T$ is said admissible if it satisfies the following conditions.

(1) If an edge $a_{i} \in E(T)$ is incident to a vertex $a_{j} \in V(T)$, then $|i-j| \equiv 1$ $(\bmod k)$.

(2) If two edges $a_{i}, a_{j} \in E(T)$ meet in a vertex $x \notin V(T)$, then $|i-j| \equiv 1$ $(\bmod k)$.

A graph is called $k$-vertex-edge-ordered if for every admissible sequence $T=$ $\left(a_{0}, \ldots, a_{k-1}\right)$ of $k$ distinct vertices and/or edges there is a $T$-cycle.

Theorem 1.1. Let $G$ be a chordal graph on at least $2 k-2$ vertices with $k \geq 3$. Then the following two statements are equivalent:

(a) $G$ is $(2 k-3)$-connected.

(b) $G$ is k-vertex-edge-ordered.

Theorem 1.1 implies a conjecture of Faudree [4] for the special case of chordal graphs.

We further generalize this concept. An orientation of an edge $e=\{u, v\}$ is a pair $(u, v) ; u$ is called the tail and $v$ the head. Let $\left(a_{0}, \ldots, a_{k-1}\right)$ be an 
admissible sequence of $k$ distinct vertices and/or edges. An orientation of the edges in this sequence is admissible if it satisfies the following conditions.

(3) If the vertex $a_{i}$ is the tail of the edge $a_{j}$, then $i \equiv j-1(\bmod k)$.

(4) If the vertex $a_{i}$ is the head of the edge $a_{j}$, then $i \equiv j+1(\bmod k)$.

(5) If two edges $a_{i}, a_{j} \in E(T)$ meet in a vertex $x \notin V(T)$ and $j \equiv i+$ $1(\bmod k)$, then $x$ is the head of $a_{i}$ and the tail of $a_{j}$.

A graph is called strongly $k$-vertex-edge-ordered if for every admissible sequence $T=\left(a_{0}, \ldots, a_{k-1}\right)$ of $k$ distinct vertices and/or edges and every admissible orientation of the edges of this sequence there is a cycle $C$ of $G$ that can be traversed such that $a_{0}, \ldots, a_{k-1}$ are encountered in the given order and every edge is traversed according to its orientation, i.e., from tail to head. Clearly, $C$ is a $T$-cycle.

Theorem 1.2. Let $G$ be a chordal graph on at least $2 k$ vertices. Then the following three statements are equivalent:

(a) $G$ is $(2 k-1)$-connected.

(b) $G$ is k-linked.

(c) $G$ is strongly k-vertex-edge-ordered.

\section{ProOFs}

Let $G$ be a graph and let $x$ be a vertex of $G$. Then $N(x)$ denotes the set of all vertices adjacent to $x$ in $G$. A vertex $x$ of a graph $G$ is simplicial if the subgraph $G[N(x)]$ of $G$ induced by $N(x)$ is complete. The following Proposition 2.1 is a consequence of a well-known theorem of Dirac [3].

Proposition 2.1. Let $G$ be a k-connected chordal graph. Then the following hold:

(a) There is a simplicial vertex $x \in V(G)$, and $G-x$ is chordal.

(b) If $G$ is not complete and $x$ is a simplicial vertex of $G$, then $G-x$ is $k$-connected.

The following Proposition 2.2 will be frequently used in the proof of Theorem 1.1. Its easy proof is left to the reader.

Proposition 2.2. Let $G$ be a graph, $T=\left(a_{0}, \ldots, a_{k-1}\right)$ be an admissible sequence of distinct vertices and/or edges, $X \subseteq V(G)$, and $J \subseteq\{0, \ldots, k-1\}$. 
If for every vertex $x \in X$ there is a $j \in J$ such that either $x=a_{j}$ or $x$ is incident to the edge $a_{j}$, then $|X| \leq 2|J|$.

Proof of Theorem 1.1. To show that $(a)$ implies (b), we apply induction on $|G|$. Let $T=\left(a_{0}, \ldots, a_{k-1}\right)$ be an admissible sequence. If $G$ is complete the statement of the theorem is clearly true. Hence we may assume that $G$ is not complete and, therefore, $|G| \geq 2 k-1$. By Proposition 2.1, there is a simplicial vertex $u \in V(G)$ and $G-u$ is $(2 k-3)$-connected and chordal. Note that $|N(u)| \geq 2 k-3$. Let $H=G[N(u) \cup\{u\}]$. Clearly, $H$ is complete. Consequently, the assertion is true if $W(T) \subseteq V(H)$. So, we henceforth assume that

$$
W(T) \nsubseteq \unrhd(H)
$$

If $u \notin W(T)$, then we apply the induction hypothesis to $G-u$, and we are done. If $u \in W(T)$, then we construct an admissible sequence $T^{\prime}=$ $\left(a_{0}^{\prime}, \ldots, a_{k-1}^{\prime}\right)$ of vertices and/or edges of $G-u$. Hence, by the induction hypothesis, there is a $T^{\prime}$-cycle $C^{\prime}$ in $G-u$. It is easy to see that $C^{\prime}$ can be extended to a $T$-cycle $C$ in $G$. For the construction of $T^{\prime}$ we distinguish the following cases.

Case 1. $u \in V(T)$, say $u=a_{0}$.

Case 1.1. $u$ is incident to an edge in $T$, say $a_{1}$.

By Proposition 2.2 and (1), $N(u) \backslash W(T) \neq \emptyset$. Let $v \in N(u)$ be the end of $a_{1}$ and $w \in N(u) \backslash W(T)$. Put $a_{0}^{\prime}=w, a_{1}^{\prime}=\{v, w\}$, and $a_{i}^{\prime}=a_{i}$ for $i \in\{2, \ldots, k-2\}$. If $a_{k-1}$ is an edge incident to $u$, then let $x \in N(u)$ be the end of $a_{k-1}$ and put $a_{k-1}^{\prime}=\{w, x\}$. Otherwise, let $a_{k-1}^{\prime}=a_{k-1}$.

Case 1.2. $u$ is not incident with any edge in $T$.

If $|N(u) \backslash W(T)| \geq 2$, then let $v, w \in N(u) \backslash W(T)$, and put $a_{0}^{\prime}=\{v, w\}$ and $a_{i}^{\prime}=a_{i}$ for all $i \in\{1, \ldots, k-1\}$. If $|N(u) \backslash W(T)| \leq 1$, then there is a vertex $v \in N(u)$ such that either $v=a_{j}$ or $v$ is incident to the edge $a_{j}$ and to no other edge in $T$ where $j \in\{1, k-1\}$. If not, then all vertices but at most one in $N(u)$ are either in $V(T) \backslash\left\{a_{0}, a_{1}, a_{k-1}\right\}$ or incident to an edge in $E(T) \backslash\left\{a_{1}, a_{k-1}\right\}$. By Proposition 2.2 this implies that $|N(u)|-1 \leq$ $2(k-3)<2 k-4$, contradicting $|N(u)| \geq 2 k-3$. W.l.o.g., we may assume that $j=1$. If $|N(u) \backslash W(T)|=1$, then let $w \in N(u) \backslash W(T)$ and put $a_{0}^{\prime}=\{v, w\}$ and $a_{i}^{\prime}=a_{i}$ for all $i \in\{1, \ldots, k-1\}$. If $|N(u) \backslash W(T)|=0$, 
then $a_{1}$ is an edge. If not, then $a_{1}=v$ and therefore, by Proposition 2.2, $W(T)=N(u) \cup\{u\}$, contradicting (1). In a similar way it can be shown that there is a vertex $w \in N(u) \backslash\{v\}$ such that either $w=a_{k-1}$ or $w$ is incident to the edge $a_{k-1}$ and to no other edge in $T$. Put $a_{0}^{\prime}=\{v, w\}$ and $a_{i}^{\prime}=a_{i}$ for all $i \in\{1, \ldots, k-1\}$.

Case 2. $u \notin V(T)$.

Case 2.1. $u$ is incident to two edges in $T$, say to $a_{0}, a k-1$.

Let $v \in N(u)$ be the end of $a_{0}$, and $w \in N(u)$ be the end of $a_{k-1}$. If $|N(u) \backslash W(T)| \geq 1$, then let $x \in N(u) \backslash W(T)$, and put $a_{0}^{\prime}=\{v, x\}, a_{k-1}^{\prime}=$ $\{x, w\}$, and $a_{i}^{\prime}=a_{i}$ for $i \in\{1, \ldots, k-2\}$. If $|N(u) \backslash W(T)|=0$, then, by Proposition 2.2, $v \neq a_{1}$ and $w \neq a_{k-2}$. Put $a_{0}^{\prime}=v, a_{k-1}^{\prime}=\{v, w\}$ and $a_{i}^{\prime}=a_{i}$ for $i \in\{1, \ldots, k-2\}$.

Case 2.2. $u$ is incident to exactly one edge in $T$, say to $a_{0}$.

Let $v \in N(u)$ be the end of $a_{0}$. If $|N(u) \backslash W(T)| \geq 1$, then let $w \in$ $N(u) \backslash W(T)$ and put $a_{0}^{\prime}=\{v, w\}$ and $a_{i}^{\prime}=a_{i}$ for $i \in\{1, \ldots, k-1\}$. If $|N(u) \backslash W(T)|=0$, then it follows by Proposition 2.2 and (1) that $v \neq a_{1}$ and $v \neq a_{k-1}$. By the essentially the same arguments as in Case 1.2 it follows, that if $v \notin V(T)$ and $v$ is not incident to any edge in $E(T) \backslash\left\{a_{0}\right\}$, then there is a vertex $w \in N(u) \backslash\{v\}$ such that either $w=a_{j}$ or $w$ is incident to the edge $a_{j}$ and to no other edge in $T$ where $j \in\{1, k-1\}$. We may assume w.l.o.g. that $j=1$. Put $a_{0}^{\prime}=\{v, w\}$ and $a_{i}^{\prime}=a_{i}$ for $i \in\{1, \ldots, k-1\}$. If $v$ is incident to an edge in $E(T) \backslash\left\{a_{0}\right\}$, say $a_{1}$, then there is a vertex $w$ such that either $w=a_{k-1}$ or $w$ is incident to the edge $a_{k-1}$ and to no other edge in $T$. Put $a_{0}^{\prime}=\{v, w\}$ and $a_{i}^{\prime}=a_{i}$ for $i \in\{1, \ldots, k-1\}$.

Next, we prove that $(b)$ implies $(a)$. It is clear that every $k$-vertex-edgeordered graph is connected. Let $G$ be a connected chordal graph on at least $2 k-2$ vertices that is not $(2 k-3)$-connected. $G$ has a minimal separator $S \subseteq V(G)$ with $|S| \leq 2 k-4$. Let $G_{1}, G_{2}$ be two distinct components of $G-S$. Since $G$ is chordal, the subgraph $H$ of $G$ induced by $S$ is complete. Let $Z=\left\{a_{1}, \ldots, a_{r-2}\right\}$ be a collection of vertices and/or edges in $H$ such that $Z$ is a perfect matching of $H$ if $|H|$ is even and a maximal matching plus the (only) unsaturated vertex, otherwise. Note that $r \leq k$. Let $T=$ $\left(a_{0}, \ldots, a_{r-1}\right)$ where $a_{0} \in V\left(G_{1}\right)$ and $a_{r-1} \in V\left(G_{2}\right)$. It is not hard to see that there is no $T$-cycle in $G$. Hence every $k$-vertex-edge-ordered chordal graph with at least $2 k-2$ vertices is $(2 k-3)$-connected. 
Proof of Theorem 1.2. To show that (a) implies (b), we apply induction on $|G|$. Since $G$ is $(2 k-1)$-connected, $|G| \geq 2 k$. If $|G|=2 k$, then $G$ is complete, and hence it is $k$-linked. If $|G|>2 k$, then it follows from Proposition 2.1 that $G$ has a simplicial vertex $x$ and $G-x$ is $(2 k-1)$-connected and chordal. Let $L=\left\{\left(s_{0}, t_{0}\right), \ldots,\left(s_{k-1}, t_{k-1}\right)\right\}$ be a set of $k$ distinct pairs of vertices of $G$. Let $l$ denote the number of pairs in $L$ containing $x$. If $l=0$, we apply the induction hypothesis to $G-x$, and we are done. We may therefore assume that $l \geq 1$, say $x=s_{0}=\ldots=s_{l-1}$. Let $A=\left\{t_{0}, \ldots, t_{l-1}\right\}$, and suppose that $A^{\prime}=\left\{t_{0}, \ldots, t_{m-1}\right\}=A \cap N(x)$. If there is a $t_{i} \in A$ such that $t_{i}=x$, then suppose that $i=l-1$. Consequently, $A^{\prime \prime}=A \backslash\left(A^{\prime} \cup\{x\}\right)=$ $\left\{t_{m}, \ldots, t_{n-1}\right\}$ where $n=l-1$ if $x=t_{l-1}$ and $n=l$, otherwise. Since $|N(x)| \geq 2 k-1, \mid N(x) \backslash\left(A^{\prime} \cup\left\{s_{l}, \ldots, s_{k-1}, t_{l}, \ldots, t_{k-1}\right\} \mid \geq 2 k-1-m-\right.$ $2(k-l)=2 l-m-1 \geq l-m$. Consequently, there is a subset $B \subseteq N(x) \backslash\left(A^{\prime} \cup\right.$ $\left\{s_{l}, \ldots, s_{k-1}, t_{l}, \ldots, t_{k-1}\right\}$ such that $|B| \geq l-m$. Let $B=\left\{y_{m}, \ldots, y_{n-1}\right\}$, and let $B^{\prime}=A^{\prime} \cup B$. It follows from the induction hypothesis, that $G-x$ contains pairwise disjoint paths $Q_{0}, \ldots, Q_{n-1}, P_{l}, \ldots, P_{k-1}$ such that $Q_{i}$ is the trivial path consisting of $t_{i}$ for $i \in\{0, \ldots, m-1\}, Q_{i}$ links $y_{i}$ to $t_{i}$ for $i \in\{m, \ldots, n-1\}$, and $P_{i}$ links $s_{i}$ to $t_{i}$ for $i \in\{l, \ldots, k-1\}$. For $i \in\{0, \ldots, n-1\}$ let $P_{i}$ be the path obtained from $Q_{i}$ by adding the edge $\left\{y_{i}, x\right\}$. If $t_{l-1}=x$, let $P_{l-1}$ be the trivial path consisting of $x$. Obviously, the paths $P_{0}, \ldots, P_{k-1}$ form the desired $L$-linkage in $G$.

Next, we prove that $(b)$ implies $(c)$. Let $G$ be $k$-linked and let $T=$ $\left(a_{0}, \ldots, a_{k-1}\right)$ be an admissible sequence together with an admissible orientation of the edges. A vertex in $V(T)$ is said to be isolated if it is not incident with any edge in $E(T)$. Let $M$ denote the set of all isolated vertices in $V(T)$, and let $T^{\prime}=\left(a_{i_{0}}, \ldots, a_{i_{r-1}}\right)$ be the subsequence of $T$ obtained by deleting all elements $a_{i} \in V(T) \backslash M$. For $e \in E(T)$ let $s(e)$ and $t(e)$ denote the head and the tail of $e$, respectively, and set $s(x)=t(x)=x$ for all $x \in M$. Let $L=\left\{\left(s_{0}, t_{0}\right), \ldots,\left(s_{r-1}, t_{r-1}\right)\right\}$ where $s_{j}=s\left(a_{i_{j}}\right)$ for $0 \leq j \leq r-1$, $t_{j}=t\left(a_{i_{j+1}}\right)$ for $0 \leq j \leq r-2$, and $t_{r-1}=t\left(a_{i_{0}}\right)$. Since $G$ is $k$-linked there is an $L$-linkage, and it is not hard to see that the union of an $L$-linkage and $E(T)$ forms the desired cycle.

Eventually, we prove that $(c)$ implies $(a)$ It is clear that every strongly $k$ vertex-edge-ordered graph is connected. Let $G$ be a connected chordal graph on at least $2 k$ vertices that is not $(2 k-1)$-connected. $G$ has a minimal separator $S \subseteq V(G)$ with $r=|S| \leq 2 k-2$. Let $G_{1}, G_{2}$ be two distinct components of $G-S$. Since $G$ is chordal, the subgraph $H$ of $G$ induced by $S$ is complete. Let $Q=v_{1}, \ldots, v_{r}$ be a Hamiltonian path of $H$, and let 
$u_{1}$ and $u_{2}$ be vertices of $G_{1}$ and $G_{2}$, respectively, such that $u_{1}$ is adjacent to $v_{1}$ and $u_{2}$ is adjacent to $v_{r}$ in $G$. For $1 \leq i \leq\left\lfloor\frac{r-1}{2}\right\rfloor$, let $e_{i}$ denote the oriented edge $\left(v_{2 i}, v_{2 i+1}\right)$. Furthermore, let $e_{0}$ and $e_{\left\lfloor\frac{r-1}{2}\right\rfloor+1}$ denote the oriented edges $\left(u_{1}, v_{1}\right)$ and $\left(v_{r}, u_{2}\right)$, respectively. It is not hard to see, that $G$ does not contain a cycle that can be traversed such that $e_{0}, \ldots, e_{\left\lfloor\frac{r-1}{2}\right\rfloor+1}$ are encountered in the given order and every edge is traversed according to its orientation. Since $\left\lfloor\frac{r-1}{2}\right\rfloor+1 \leq k$, this shows that $G$ is not strongly $k$-vertex-edge-ordered. Hence every strongly $k$-vertex-edge-ordered chordal graph is $(2 k-1)$-connected.

\section{REFERENCES}

[1] B. Bollobás and A. Thomason, Highly linked graphs, Combinatorica 16 (1996) $313-320$.

[2] R. Diestel, Graph Theory, Graduate Texts in Mathematics 173 (Springer, 2000).

[3] G.A. Dirac, On rigid circuit graphs, Abh. Math. Sem. Univ. Hamburg 25 (1961) $71-76$.

[4] R.J. Faudree, Survey on results on k-ordered graphs, Discrete Math. 229 (2001) $73-87$.

[5] H.A. Jung, Eine veralgemeinerung des $n$-fachen zusammenhangs für graphen, Math. Ann. 187 (1970) 95-103.

[6] D.G. Larman and P. Mani, On the existence of certain configurations within graphs and the 1-skeleton of polytopes, Proc. London Math. Soc. 20 (1970) 144-160.

[7] L. Ng and M. Schultz, k-ordered Hamiltonian graphs, J. Graph Theory 24 (1997) 45-57.

[8] R. Thomas and P. Wollan, An improved linear edge bound for graph linkages, to appear in European J. Comb.

Received 20 September 2007

Revised 1 April 2008

Accepted 2 April 2008 